\title{
Smooth metric measure spaces with non-negative curvature
}

\author{
Ovidiu Munteanu And Jiaping Wang
}

\begin{abstract}
In this paper, we study both function theoretic and spectral properties on complete non-compact smooth metric measure space $(M, g$, $\left.\mathrm{e}^{-f} d v\right)$ with non-negative Bakry-Émery Ricci curvature. Among other things, we derive a gradient estimate for positive $f$-harmonic functions and obtain as a consequence the strong Liouville property under the optimal sublinear growth assumption on $f$. We also establish a sharp upper bound of the bottom spectrum of the $f$-Laplacian in terms of the linear growth rate of $f$. Moreover, we show that if equality holds and $M$ is not connected at infinity, then $M$ must be a cylinder. As an application, we conclude steady Ricci solitons must be connected at infinity.
\end{abstract}

\section{Introduction}

On a Riemannian manifold $(M, g)$, the consideration of weighted measure of the form $\mathrm{e}^{-f} d v$, where $f$ is a smooth function and $d v$ is the volume element induced by the metric $g$, arises naturally in various situations. It can be viewed as the volume form of a suitable conformal change of the metric $g$. Perhaps a more notable example is in the work of Perelman [27], where he introduces a functional involving the integral of the scalar curvature with respect to such a weighted measure and formulates the Ricci flow as the gradient flow of the functional. The triple $\left(M, g, \mathrm{e}^{-f} d v\right)$ is customarily called a smooth metric measure space. The differential operator $\Delta_{f}$, which is called $f$-Laplacian and given by

$$
\Delta_{f}:=\Delta-\nabla f \cdot \nabla
$$

is more naturally associated with such a smooth metric measure space than the classical Laplacian as it is symmetric with respect to the measure $\mathrm{e}^{-f} d v$. 
That is,

$$
\int_{M}\langle\nabla \varphi, \nabla \psi\rangle \mathrm{e}^{-f}=-\int_{M}\left(\Delta_{f} \varphi\right) \psi \mathrm{e}^{-f},
$$

for any $\varphi, \psi \in C_{0}^{\infty}(M)$.

Again, we point out that the operator $f$-Laplacian is very much related to the Laplacian of a suitable conformal change of the background Riemannian metric. It also appears as the generator of a class of stochastic diffusion processes, the Brownian motion with drifts.

The Bakry-Émery Ricci tensor [1] of the metric measure space $\left(M, g, \mathrm{e}^{-f}\right.$ $d v)$ is defined by

$$
\operatorname{Ric}_{f}:=\operatorname{Ric}+\operatorname{Hess}(f),
$$

where Ric denotes the Ricci curvature of $M$ and Hess $(f)$ the Hessian of $f$. This curvature relates to $f$-Laplacian via the following Bochner formula:

$$
\Delta_{f}|\nabla u|^{2}=2|\operatorname{Hess}(u)|^{2}+2\left\langle\nabla u, \nabla \Delta_{f} u\right\rangle+2 \operatorname{Ric}_{f}(\nabla u, \nabla u)
$$

This of course suggests the important role of $\mathrm{Ric}_{f}$ in the analysis of $f$-Laplacian. Perhaps as a more prominent example, $\mathrm{Ric}_{f}$ also appears in the study of the Ricci flow. The gradient solitons of the Ricci flow, which arise from the singularity analysis of the Ricci flow, are defined to be complete manifolds $(M, g)$ that the following equation

$$
\operatorname{Ric}_{f}=\lambda g
$$

holds for some function $f$ and constant $\lambda$. Obviously, the Einstein manifolds are gradient Ricci solitons. The gradient Ricci solitons are called shrinking, steady and expanding accordingly when $\lambda>0, \lambda=0$ and $\lambda<0,[15]$.

The classification of gradient Ricci solitons is an important problem from the point of view of both the Ricci flow singularity analysis and purely as a class of geometric partial differential equations. The problem has received much attention recently. The book [7] is a good source for some of the important results. But it seems fair to say that the whole picture is far from clear for now.

Partially motivated by interest in the study of gradient Ricci solitons, various attempts have been made recently to study the geometry and analysis on general metric measure spaces. We will refer the readers to $[22,31]$ for some of the results. It should be noted however a while back, Lichnerowicz [23] has already done some pioneering work in this direction. In 
particular, he has extended the classical Cheeger-Gromoll splitting theorem to the metric measure spaces with $\operatorname{Ric}_{f} \geq 0$ and $f$ bounded.

In this paper, we will investigate some function theoretic and spectral properties of metric measure space $\left(M, g, \mathrm{e}^{-f} d v\right)$. While the results are of independent interest, applications to the steady gradient Ricci solitons are no doubt of our main focus. Throughout, we will assume $\operatorname{Ric}_{f} \geq 0$ and $f$ is of linear growth unless otherwise noted. Recall that $f$ is of linear growth if for all $x$ in $M$,

$$
|f|(x) \leq \alpha r(x)+\beta
$$

for some constants $\alpha$ and $\beta$, where $r(x):=d(p, x)$ is the geodesic distance function to a fixed point $p$ in $M$. The linear growth rate $a$ of $f$ is then defined as the infimum of all such values $\alpha$.

Clearly, a steady gradient Ricci soliton $(M, g)$ satisfies $\operatorname{Ric}_{f}=0$ for some $f$. It is also well-known that the potential function $f$ in this case is of linear growth. So all of our results are applicable to the steady gradient solitons. On the other hand, it should also be noted that many, if not all, of our results will fail without the growth assumption on function $f$.

Our first result gives a gradient estimate for positive $f$-harmonic functions on $\left(M, g, \mathrm{e}^{-f} d v\right)$.

Theorem 1.1. Let $\left(M, g, e^{-f} d v\right)$ be a complete non-compact smooth metric measure space with Ric $_{f} \geq 0$. Assume that $f$ has linear growth rate a and let $u>0$ be $f$-harmonic on $M$, i.e., $\Delta_{f} u=0$ on $M$. Then the following gradient estimate holds true on $M$ :

$$
|\nabla \log u| \leq C(n) a,
$$

where the constant $C(n)$ depends only on the dimension $n$. In particular, if $f$ is of sublinear growth, then any positive $f$-harmonic function $u$ on $M$ must be constant.

This estimate is sharp as demonstrated by the following simple example.

Example 1.1 [26]. Let $M=\mathbb{R} \times \mathbb{S}^{n-1}$ and $f(t, \theta)=$ at for $t \in \mathbb{R}$ and $\theta \in$ $\mathbb{S}^{n-1}$. Then $u(t, \theta):=\mathrm{e}^{a t}$ is positive $f$-harmonic on $M$. Clearly, the linear growth rate of $f$ is $a$ and $|\nabla \log u|=a$.

Although the statement in the theorem takes the form of Yau's classical result on the positive harmonic functions, we would like to point out that our proof is quite different. In the classical case of the Ricci curvature, 
Yau [34] directly works with $\log u$ and obtains an estimate on $|\nabla \log u|$ via the Bochner formula. This approach works also with the $N$-Bakry-Émery Ricci curvature given by

$$
\operatorname{Ric}_{f}^{N}:=\operatorname{Ric}+\operatorname{Hess}_{f}-\frac{1}{N} d f \otimes d f
$$

as demonstrated by $\mathrm{Li}[22]$. For the case of curvature $\operatorname{Ric}_{f}$, if one imposes suitable assumption on $|\nabla f|$, say, it is bounded, then it is still possible to utilize Yau's argument as shown by Wu [32]. However, with only the growth assumption on $f$, this direct approach seems to run into essential obstacles. Our argument relies on both Yau's idea and the well-known De GiorgiNash-Moser theory. In a recent paper [2], Brighton, by applying Yau's idea to function $u^{\epsilon}$ instead of $\log u$, proved bounded $f$-harmonic functions must be constant without any assumption on $f$ so long as $\operatorname{Ric}_{f} \geq 0$. In our proof, we first refined Brighton's argument and derived the gradient estimate under the assumption that $u$ is of exponential growth. Here, no growth assumption on $f$ is necessary. In particular, this implies that any sub-exponential growth positive $f$-harmonic on $M$ with $\operatorname{Ric}_{f} \geq 0$ is constant. The growth assumption on $f$ was then used to get the desired growth control on $u$. For that, we utilize a different set of techniques including a mean value inequality obtained through the Moser's iteration argument.

We also deal with the polynomial growth $f$-harmonic functions. Here, the result is very much parallel to the case of harmonic functions on a manifold with non-negative Ricci curvature, obtained by Cheng and Yau [10], Li-Tam [18], Li [17] and Colding-Minicozzi [11], respectively. We define the space

$$
\mathcal{H}^{d}(M):=\left\{u: \Delta_{f} u=0 \quad \text { and } \quad|u|(x) \leq C(r(x)+1)^{d}\right\}
$$

Theorem 1.2. Let $\left(M, g, e^{-f} d v\right)$ be a complete non-compact smooth metric measure space with Ric $_{f} \geq 0$ and $f$ bounded. Then we have

$$
\begin{aligned}
& \operatorname{dim} \mathcal{H}^{d}(M)=1, \quad \text { if } d<1 \\
& \operatorname{dim} \mathcal{H}^{d}(M) \leq n+1, \quad \text { if } d=1
\end{aligned}
$$

and

$$
\operatorname{dim} \mathcal{H}^{d}(M) \leq C d^{n-1}, \quad \text { if } d \geq 1
$$

Our second objective is to study the spectrum of the $f$-Laplacian on $\left(M, g, \mathrm{e}^{-f} d v\right)$. Define $\lambda_{1}\left(\Delta_{f}\right):=\min \operatorname{Spec}\left(-\Delta_{f}\right)$ to be the bottom spectrum 
of $\Delta_{f}$. By the variational characterization, we have

$$
\lambda_{1}\left(\Delta_{f}\right)=\inf _{\phi \in C_{0}^{\infty}(M)} \frac{\int_{M}|\nabla \phi|^{2} \mathrm{e}^{-f}}{\int_{M} \phi^{2} \mathrm{e}^{-f}} .
$$

The following result summarizes what we proved concerning the bottom spectrum.

Theorem 1.3. Let $\left(M, g, e^{-f} d v\right)$ be a complete non-compact smooth metric measure space with Ric $_{f} \geq 0$. Then

$$
\lambda_{1}\left(\Delta_{f}\right) \leq \frac{1}{4} a^{2},
$$

where $a$ is the linear growth rate of $f$. Moreover, if $\lambda_{1}\left(\Delta_{f}\right)=\frac{1}{4} a^{2}$, then $M$ is connected at infinity; or $M$ is isometric to $\mathbb{R} \times N$ for some compact manifold $N$.

Note that the splitting case in the theorem does occur. Indeed, for $M=$ $\mathbb{R} \times N$, if we take $f(t, y)=a t$ for $(t, y) \in \mathbb{R} \times N$, then $|\nabla f|=a, \Delta_{f} \mathrm{e}^{\frac{1}{2} a t}=$ $-\frac{1}{4} a^{2} \mathrm{e}^{\frac{1}{2} a t}$ and $\lambda_{1}\left(\Delta_{f}\right)=\frac{1}{4} a^{2}$.

Applying the preceding result to the gradient steady Ricci solitons, we obtain the following.

Corollary 1.1. A non-trivial gradient steady Ricci soliton must be connected at infinity.

This is because for a steady gradient Ricci soliton $(M, g, f)$ such that $\operatorname{Ric}_{f}=0$ on $M$ and $\sup |\nabla f|=a$, one can show $\lambda_{1}\left(\Delta_{f}\right)=\frac{1}{4} a^{2}$. Note now that the splitting case cannot arise as otherwise $\operatorname{Hess}(f)=0$ and $M$ would be Ricci flat. We remark that previous attempts on the issue of connectedness at infinity for steady solitons can be found in [12, 24].

Historically, Cheng [9] proved a sharp upper bound of the bottom spectrum of the Laplacian on a complete manifold with Ricci curvature bounded below. Later on, in $[19,20], \mathrm{Li}$ and the second author studied the rigidity issue when the sharp upper bound is achieved. Our results here are very much in the same spirit. Our arguments, however, follow [21] more closely.

The paper is organized as follows. In Section 2, we discuss Laplacian and volume comparison estimates and establish the upper bound estimate for the bottom spectrum $\lambda_{1}\left(\Delta_{f}\right)$. In Section 3, we prove the gradient estimate for positive $f$-harmonic functions and related Liouville type results concerning 
$f$-harmonic functions of polynomial growth. Finally, in Section 4 we discuss the structure of manifolds with maximal bottom spectrum and the resulting application to the steady Ricci solitons.

In a sequel to this paper [25], we will address some similar issues on smooth metric measure spaces with Ric $f$ bounded below. Among other things, we obtain a gradient estimate as Theorem 1.1 of the form $|\nabla \log u| \leq$ $c(n, a)$ if $\operatorname{Ric}_{f} \geq-(n-1)$, where $a$ now is the maximum oscillation of $f$ over all unit balls in $M$. A parallel result to Theorem 1.3 also holds true. More precisely, the bottom spectrum satisfies $\lambda_{1}\left(\Delta_{f}\right) \leq \frac{1}{4}(n-1+a)^{2}$ if $\operatorname{Ric}_{f} \geq-(n-1)$, where $a$ again is the linear growth rate of $f$. Under slightly stronger assumption on $f$ that its gradient is bounded by $a$, then that the equality holds implies the manifold $M$ must be connected at infinity unless it is a product $\mathbb{R} \times N$ with $N$ being compact.

\section{Volume comparison theorems}

In this section, we discuss Laplacian and volume comparison estimates for smooth metric measure spaces with non-negative Bakry-Émery Ricci curvature. The estimates in this section are instrumental in proving other results of this paper. Also, as an immediate application, we obtain upper bound estimates for the bottom spectrum of $\Delta_{f}$.

Let $\left(M, g, \mathrm{e}^{-f} d v\right)$ be a smooth metric measure space. Take any point $x \in M$ and denote the volume form in geodesic polar coordinates centered at $x$ with

$$
\left.d V\right|_{\exp _{x}(r \xi)}=J(x, r, \xi) d r d \xi
$$

where $r>0$ and $\xi \in S_{x} M$, a unit tangent vector at $x$. It is well-known that if $y \in M$ is any point such that $y=\exp _{x}(r \xi)$, then

$$
\Delta d(x, y)=\frac{J^{\prime}(x, r, \xi)}{J(x, r, \xi)} \quad \text { and } \quad \Delta_{f} d(x, y)=\frac{J_{f}^{\prime}(x, r, \xi)}{J_{f}(x, r, \xi)}
$$

where $J_{f}(x, r, \xi):=\mathrm{e}^{-f} J(x, r, \xi)$ is the $f$-volume form in geodesic polar coordinates. For a fixed point $p \in M$ and $R>0$, define

$$
A(R):=\sup _{x \in B_{p}(3 R)}|f|(x) .
$$

For a set $\Omega$, we will denote by $V(\Omega)$ the volume of $\Omega$ with respect to the usual volume form $d v$, and $V_{f}(\Omega)$ the $f$-volume of $\Omega$. The following result has been established in [33]. 
Lemma 2.1. Let $\left(M, g, e^{-f} d v\right)$ be a smooth metric measure space with Ric $_{f} \geq 0$. Then along any minimizing geodesic starting from $x \in B_{p}(R)$ we have

$$
\frac{J_{f}\left(x, r_{2}, \xi\right)}{J_{f}\left(x, r_{1}, \xi\right)} \leq e^{4 A}\left(\frac{r_{2}}{r_{1}}\right)^{n-1} \quad \text { and } \quad \frac{J\left(x, r_{2}, \xi\right)}{J\left(x, r_{1}, \xi\right)} \leq e^{6 A}\left(\frac{r_{2}}{r_{1}}\right)^{n-1}
$$

for any $0<r_{1}<r_{2}<R$. In particular, for any $0<r_{1}<r_{2}<R$,

$$
\frac{V_{f}\left(B_{x}\left(r_{2}\right)\right)}{V_{f}\left(B_{x}\left(r_{1}\right)\right)} \leq e^{4 A}\left(\frac{r_{2}}{r_{1}}\right)^{n} \quad \text { and } \quad \frac{V\left(B_{x}\left(r_{2}\right)\right)}{V\left(B_{x}\left(r_{1}\right)\right)} \leq e^{6 A}\left(\frac{r_{2}}{r_{1}}\right)^{n} .
$$

Here, $A=A(R)$ as defined by (2.1).

Proof of Lemma 2.1. We include a proof here for the reader's convenience. Some of the ingredients of this proof will also be used later.

Let $\gamma$ be the minimizing geodesic from $x$ to $y$ such that $\gamma(0)=x$ and $\gamma(r)=y$. Recall the following Laplace comparison theorem [12, 31],

$$
\Delta_{f} d(x, y) \leq \frac{n-1}{r}-\frac{2}{r^{2}} \int_{0}^{r} t f^{\prime}(t) d t,
$$

where $f(t):=f(\gamma(t))$. Integrating by parts, we get

$$
\Delta_{f} d(x, y) \leq \frac{n-1}{r}-\frac{2}{r} f(r)+\frac{2}{r^{2}} \int_{0}^{r} f(t) d t .
$$

For $0<r_{1}<r_{2}<R$, integrating (2.3) from $r_{1}$ to $r_{2}$ yields

$$
\begin{aligned}
\log \left(\frac{J_{f}\left(x, r_{2}, \xi\right)}{J_{f}\left(x, r_{1}, \xi\right)}\right) \leq & (n-1) \log \left(\frac{r_{2}}{r_{1}}\right)+2 \int_{r_{1}}^{r_{2}} \frac{1}{r^{2}}\left(\int_{0}^{r} f(t) d t\right) d r \\
& -2 \int_{r_{1}}^{r_{2}} \frac{1}{r} f(r) d r
\end{aligned}
$$

However,

$$
\int_{r_{1}}^{r_{2}} \frac{1}{r^{2}}\left(\int_{0}^{r} f(t) d t\right) d r=-\left.\frac{1}{r}\left(\int_{0}^{r} f(t) d t\right)\right|_{r_{1}} ^{r_{2}}+\int_{r_{1}}^{r_{2}} \frac{1}{r} f(r) d r .
$$

Plugging into (2.4), we conclude

$$
\frac{J_{f}\left(x, r_{2}, \xi\right)}{J_{f}\left(x, r_{1}, \xi\right)} \leq\left(\frac{r_{2}}{r_{1}}\right)^{n-1} \exp \left(\frac{2}{r_{1}} \int_{0}^{r_{1}} f(t) d t-\frac{2}{r_{2}} \int_{0}^{r_{2}} f(t) d t\right) .
$$


Therefore,

$$
\frac{J_{f}\left(x, r_{2}, \xi\right)}{J_{f}\left(x, r_{1}, \xi\right)} \leq \mathrm{e}^{4 A}\left(\frac{r_{2}}{r_{1}}\right)^{n-1}
$$

for all $x \in B_{p}(R)$ and $0<r_{1}<r_{2}<R$. Clearly, the corresponding result for $J(x, r, \xi)$ follows by using again the definition (2.1).

To establish the volume comparison, we use that

$$
\frac{J_{f}(x, t, \xi)}{J_{f}(x, s, \xi)} \leq \mathrm{e}^{4 A}\left(\frac{t}{s}\right)^{n-1}
$$

for any $0<s<r_{1}<t<r_{2}<R$. Integrating in $t$ from $r_{1}$ to $r_{2}$ and $s$ from 0 to $r_{1}$, we get

$$
\frac{V_{f}\left(B_{x}\left(r_{2}\right)\right)-V_{f}\left(B_{x}\left(r_{1}\right)\right)}{V_{f}\left(B_{x}\left(r_{1}\right)\right)} \leq \mathrm{e}^{4 A} \frac{\left(r_{2}\right)^{n}-\left(r_{1}\right)^{n}}{\left(r_{1}\right)^{n}} .
$$

This implies that

$$
\frac{V_{f}\left(B_{x}\left(r_{2}\right)\right)}{V_{f}\left(B_{x}\left(r_{1}\right)\right)} \leq \mathrm{e}^{4 A}\left(\frac{r_{2}}{r_{1}}\right)^{n}+1-\mathrm{e}^{4 A} \leq \mathrm{e}^{4 A}\left(\frac{r_{2}}{r_{1}}\right)^{n} .
$$

Now the volume comparison for $V\left(B_{x}(r)\right)$ follows directly from here. The lemma is proved.

Using the proof of Lemma 2.1 we obtain a sharp upper bound for $\lambda_{1}\left(\Delta_{f}\right)$, the bottom spectrum of $\Delta_{f}$, by assuming $f$ is of linear growth. Recall

$$
\lambda_{1}\left(\Delta_{f}\right):=\inf _{\phi \in C_{0}^{\infty}(M)} \frac{\int_{M}|\nabla \phi|^{2} \mathrm{e}^{-f}}{\int_{M} \phi^{2} \mathrm{e}^{-f}} .
$$

Theorem 2.1. Let $\left(M, g, e^{-f} d v\right)$ be a complete non-compact smooth metric measure space with Ric $_{f} \geq 0$. If there exist positive constants $a, b>0$ such that

$$
|f|(x) \leq \operatorname{ar}(x)+b \quad \text { for all } x \in M
$$

then we have the upper bound estimate

$$
\lambda_{1}\left(\Delta_{f}\right) \leq \frac{1}{4} a^{2}
$$

In particular, if $f$ has sublinear growth, then $\lambda_{1}\left(\Delta_{f}\right)=0$. 
Proof of Theorem 2.1. By setting $x=p, r_{1}=1$ and $r_{2}=R>1$ and noting $|f|(x) \leq \operatorname{ar}(x)+b$ on $M,(2.5)$ implies

$$
J_{f}(p, R, \xi) \leq C R^{n-1} \mathrm{e}^{-\frac{2}{R} \int_{0}^{R} f(t) d t} \leq C R^{n-1} \mathrm{e}^{a R}
$$

for all $R>1$. Therefore,

$$
V_{f}\left(B_{p}(R)\right) \leq C R^{n} \mathrm{e}^{a R}
$$

We now claim $\lambda_{1}\left(\Delta_{f}\right) \leq \frac{1}{4} a^{2}$. Indeed, take a cut-off $\psi$ on $B_{p}(R)$ such that $\psi=1$ on $B_{p}(R-1), \psi=0$ on $M \backslash B_{p}(R)$ and $|\nabla \psi| \leq c$. Consider $\phi(y):=$ $\mathrm{e}^{-\frac{1}{2}(a+\varepsilon) r(y)} \psi(y)$ as a test function in the variational principle for $\lambda_{1}\left(\Delta_{f}\right)$. Using the volume growth (2.6) we get immediately that $\lambda_{1}\left(\Delta_{f}\right) \leq \frac{1}{4}(a+\varepsilon)^{2}$. Since $\varepsilon>0$ is arbitrary this implies the desired estimate. The theorem is proved.

As indicated in the Introduction, the estimate in Theorem 2.1 is sharp. We now show that the bottom spectrum of steady Ricci solitons also attains this upper bound. Recall a gradient steady Ricci soliton is a manifold $(M, g, f)$ satisfying $R_{i j}+f_{i j}=0$. It is known that there exists a positive constant $a>0$ such that $|\nabla f|^{2}+S=a^{2}$, where $S$ is the scalar curvature of $M$. It is also known that $S \geq 0$ for any gradient steady Ricci soliton [4, 8]. Another useful relation is $\Delta f+S=0$, which is obtained directly from $\operatorname{Ric}_{f}=0$ by taking trace. In summary, a steady Ricci soliton satisfies the following.

$$
\begin{aligned}
|\nabla f|^{2}+S & =a^{2}, \quad \text { for some constant } a>0 \\
\Delta f+S & =0 \\
S & \geq 0 .
\end{aligned}
$$

We first recall a well-known result.

Lemma 2.2. Let $\left(M, g, e^{-f} d v\right)$ be a smooth metric measure space. If there exists a positive function $v>0$ such that $\Delta_{f} v \leq-\lambda v$ for some constant $\lambda>0$, then $\lambda_{1}\left(\Delta_{f}\right) \geq \lambda$.

Proof of Lemma 2.2. For completeness, we include a proof of this result. For an exhaustion of $M$ by compact domains $\Omega_{i} \subset \subset M$, consider the first 
Dirichlet eigenfunction $u_{i}$.

$$
\begin{aligned}
\Delta_{f} u_{i} & =-\lambda_{1}\left(\Omega_{i}\right) u_{i} \quad \text { in } \Omega_{i} \\
u_{i} & =0 \quad \text { on } \partial \Omega_{i} .
\end{aligned}
$$

It is known that we may assume $u_{i}>0$. By the strong maximum principle, $\frac{\partial u_{i}}{\partial \eta}<0$ on $\partial \Omega_{i}$. Now

$$
\begin{aligned}
\left(\lambda_{1}\left(\Omega_{i}\right)-\lambda\right) \int_{\Omega_{i}} v u_{i} \mathrm{e}^{-f} & \geq \int_{\Omega_{i}}\left(u_{i} \Delta_{f} v-v \Delta_{f} u_{i}\right) \mathrm{e}^{-f} \\
& =\int_{\partial \Omega_{i}}\left(u_{i} \frac{\partial v}{\partial \eta}-v \frac{\partial u_{i}}{\partial \eta}\right) \mathrm{e}^{-f}=-\int_{\partial \Omega_{i}} v \frac{\partial u_{i}}{\partial \eta} \mathrm{e}^{-f}>0
\end{aligned}
$$

Since both $u_{i}$ and $v$ are positive, this shows $\lambda_{1}\left(\Omega_{i}\right) \geq \lambda$. The Lemma follows from

$$
\lim _{i \rightarrow \infty} \lambda_{1}\left(\Omega_{i}\right)=\lambda_{1}\left(\Delta_{f}\right)
$$

Proposition 2.1. Let $(M, g, f)$ be a gradient steady Ricci soliton, normalized as in (2.7). Then $\lambda_{1}\left(\Delta_{f}\right)=\frac{a^{2}}{4}$.

Proof of Proposition 2.1. Since $S \geq 0$, it follows that $|\nabla f| \leq a$. Therefore, $|f|(x) \leq \operatorname{ar}(x)+b$ for any $x \in M$. So by Theorem $2.1, \lambda_{1}\left(\Delta_{f}\right) \leq \frac{1}{4} a^{2}$. To show the equality, we proceed as follows. Observe that

$$
\Delta_{f} \mathrm{e}^{\frac{1}{2} f}=\left(\frac{1}{2} \Delta_{f}(f)+\frac{1}{4}|\nabla f|^{2}\right) \mathrm{e}^{\frac{1}{2} f} .
$$

But for a steady soliton,

$$
\Delta_{f}(f)=\Delta f-|\nabla f|^{2}=-\left(S+|\nabla f|^{2}\right)=-a^{2} .
$$

Since $|\nabla f| \leq a$, we conclude that

$$
\begin{aligned}
\Delta_{f} \mathrm{e}^{\frac{1}{2} f} & =\left(-\frac{1}{2} a^{2}+\frac{1}{4}|\nabla f|^{2}\right) \mathrm{e}^{\frac{1}{2} f} \\
& \leq\left(-\frac{1}{2} a^{2}+\frac{1}{4} a^{2}\right) \mathrm{e}^{\frac{1}{2} f}=-\frac{a^{2}}{4} \mathrm{e}^{\frac{1}{2} f} .
\end{aligned}
$$

By Lemma 2.2, we have $\lambda_{1}\left(\Delta_{f}\right) \geq \frac{a^{2}}{4}$. This proves Proposition 2.1. 


\section{3. $f$-harmonic functions}

In this section, we continue to assume $\operatorname{Ric}_{f} \geq 0$ on $\left(M, g, \mathrm{e}^{-f} d v\right)$. The main objective is to derive the following global gradient estimate for positive $f$-harmonic functions defined on $M$. This in particular leads to a strong Liouville theorem under optimal growth assumption on $f$.

For a fixed point $p \in M$, we let $r(x):=d(p, x)$.

Theorem 3.1. Let $\left(M, g, e^{-f} d v\right)$ be a complete non-compact smooth metric measure space with Ric $_{f} \geq 0$. Assume there exist positive constants a and $b$ such that

$$
|f|(x) \leq a r(x)+b \quad \text { on } M \text {. }
$$

Let $u>0$ be $f$-harmonic on $M$. Then the following gradient estimate holds true on $M$ :

$$
|\nabla \log u| \leq C(n) a
$$

where constant $C(n)$ depending only on $n$, the dimension of $M$.

An immediate consequence is the following strong Liouville property. As noted by the explicit example in first section, the growth assumption on $f$ is optimal.

Corollary 3.1. Let $\left(M, g, e^{-f} d v\right)$ be a complete non-compact smooth metric measure space with Ric $_{f} \geq 0$. If $f$ is of sublinear growth, then any positive $f$-harmonic function $u$ on $M$ is constant.

We prove Theorem 3.1 in several steps. First, we show that $|\nabla \log u|$ can be controlled from above by $\frac{1}{R} \sup _{B_{p}(R)} \log (u+1)$. This follows by adapting Yau's [34] argument. We then verify that $u$ must be of exponential growth with the exponent controlled by the constant $a$. To achieve this, we use the Moser iteration technique, following the ideas in $[13,28]$.

Proposition 3.1. Let $\left(M, g, e^{-f} d v\right)$ be a complete non-compact smooth metric measure space with Ric $_{f} \geq 0$. Assume that there exist constants a $>0$ and $b>0$ such that

$$
|f|(x) \leq a r(x)+b \quad \text { on } M .
$$

Then for any positive $f$-harmonic function $u$ on $M$, we have

$$
\sup _{M}|\nabla \log u|^{2} \leq C(n)\left(\Omega(u)^{2}+a \Omega(u)\right)
$$


where $C(n)>0$ is a constant only depending on the dimension $n$ of $M$ and

$$
\Omega(u):=\limsup _{R \rightarrow \infty}\left\{\frac{1}{R} \sup _{B_{p}(R)} \log (u+1)\right\} .
$$

Proof of Proposition 3.1. The proof is based on Yau's argument using the Bochner technique, with a modification similar to that in [2]. Let $u>0$ be $f$-harmonic. For $0<\epsilon<\frac{1}{2}$, define

$$
h:=\frac{1}{\epsilon} u^{\epsilon}
$$

Then a direct computation gives $\Delta_{f} h=(\epsilon-1) u^{\epsilon-2}|\nabla u|^{2}$.

Let us denote $\sigma:=|\nabla h|^{2}=u^{2 \epsilon-2}|\nabla u|^{2}$. The Bochner formula asserts that

$$
\begin{aligned}
\frac{1}{2} \Delta_{f} \sigma & =\left|h_{i j}\right|^{2}+\left\langle\nabla h, \nabla\left(\Delta_{f} h\right)\right\rangle+\operatorname{Ric}_{f}(\nabla h, \nabla h) \\
& \geq\left\langle\nabla h, \nabla\left(\Delta_{f} h\right)\right\rangle=(\epsilon-1)\left\langle\nabla h, \nabla\left(u^{\epsilon-2}|\nabla u|^{2}\right)\right\rangle \\
& =(\epsilon-1)\left\langle\nabla h, \nabla\left(u^{-\epsilon} \sigma\right)\right\rangle .
\end{aligned}
$$

Notice that

$$
\begin{aligned}
\left\langle\nabla h, \nabla\left(u^{-\epsilon} \sigma\right)\right\rangle & =-\epsilon\langle\nabla h, \nabla u\rangle u^{-\epsilon-1} \sigma+u^{-\epsilon}\langle\nabla h, \nabla \sigma\rangle \\
& =-\epsilon|\nabla h|^{2} u^{-2 \epsilon} \sigma+u^{-\epsilon}\langle\nabla h, \nabla \sigma\rangle .
\end{aligned}
$$

Consequently,

$$
\frac{1}{2} \Delta_{f} \sigma \geq \epsilon(1-\epsilon) u^{-2 \epsilon} \sigma^{2}+(\epsilon-1) u^{-\epsilon}\langle\nabla h, \nabla \sigma\rangle
$$

We now take a function $\phi:[0,2 R] \rightarrow[0,1]$ with the following properties:

$$
\begin{aligned}
\phi & =1 \text { on }[0, R] \\
\operatorname{supp}(\phi) & \subseteq[0,2 R) \\
-\frac{c}{R} & \leq \frac{\phi^{\prime}}{\sqrt{\phi}} \leq 0 \\
\left|\phi^{\prime \prime}\right| & \leq \frac{c}{R^{2}}
\end{aligned}
$$

where $c>0$ is a universal constant. We continue to denote by $c>0$ some universal constant, which may differ from line to line. We use this function 
to define a cut-off on $M$ by taking $\phi(x):=\phi(r(x))$. Define $G:=\phi \sigma$. Then $G$ is non-negative on $M$ and has compact support in $B_{p}(2 R)$. Therefore, it achieves its maximum at some point $y \in B_{p}(2 R)$.

Without loss of generality we can assume that $y$ is not in the cut-locus of $p$. So $\phi$ is smooth at $y$. Now at point $y$, we have

$$
\Delta G(y) \leq 0 \quad \text { and } \quad \nabla G(y)=0 .
$$

By (2.3) together with $|f|(x) \leq \operatorname{ar}(x)+b$, we get

$$
\Delta_{f} r(x) \leq \frac{n-1+4 b}{r}+3 a .
$$

So there exists $r_{0}>0$ such that $\Delta_{f} r(x) \leq 4 a$ for $x \in M \backslash B_{p}\left(r_{0}\right)$. Therefore, for $R>r_{0}$, we have

$$
\Delta_{f} \phi=\phi^{\prime} \Delta_{f} r+\phi^{\prime \prime} \geq-c\left(\frac{a}{R}+\frac{1}{R^{2}}\right)
$$

and

$$
\phi^{-1}|\nabla \phi|^{2} \leq \frac{c}{R^{2}} .
$$

Combining with inequality (3.1), we find that

$$
\begin{aligned}
\frac{1}{2} \Delta_{f} G= & \frac{1}{2} \phi \Delta_{f} \sigma+\frac{1}{2} \sigma \Delta_{f} \phi+\langle\nabla \phi, \nabla \sigma\rangle \\
\geq & \epsilon(1-\epsilon) u^{-2 \epsilon} \phi^{-1} G^{2}+(\epsilon-1) u^{-\epsilon}\left\langle\nabla h, \nabla\left(\phi^{-1} G\right)\right\rangle \phi \\
& -c\left(\frac{a}{R}+\frac{1}{R^{2}}\right) \phi^{-1} G+\left\langle\nabla \phi, \nabla\left(\phi^{-1} G\right)\right\rangle \\
= & \epsilon(1-\epsilon) u^{-2 \epsilon} \phi^{-1} G^{2}+(\epsilon-1) u^{-\epsilon}\left\langle\nabla h, \nabla \phi^{-1}\right\rangle G \phi \\
& +(\epsilon-1) u^{-\epsilon}\langle\nabla h, \nabla G\rangle-c\left(\frac{a}{R}+\frac{1}{R^{2}}\right) \phi^{-1} G \\
& -|\nabla \phi|^{2} \phi^{-2} G+\phi^{-1}\langle\nabla \phi, \nabla G\rangle .
\end{aligned}
$$

After multiplying both sides by $\phi$ and invoking (3.2), we conclude that at $y$,

$$
0 \geq \epsilon(1-\epsilon) u^{-2 \epsilon} G^{2}-(1-\epsilon) u^{-\epsilon}|\nabla h||\nabla \phi| G-c\left(\frac{a}{R}+\frac{1}{R^{2}}\right) G .
$$

Note that

$$
u^{-\epsilon}|\nabla h||\nabla \phi| \leq \frac{c}{R} u^{-\epsilon} \sigma^{\frac{1}{2}} \phi^{\frac{1}{2}}=\frac{c}{R}\left(u^{-2 \epsilon} G\right)^{\frac{1}{2}}
$$


This shows at point $y$,

$$
\epsilon\left(u^{-2 \epsilon} G\right)-\frac{c}{R}\left(u^{-2 \epsilon} G\right)^{\frac{1}{2}}-c\left(\frac{a}{R}+\frac{1}{R^{2}}\right) \leq 0 .
$$

Solving this as a quadratic inequality in $\left(u^{-2 \epsilon} G\right)^{\frac{1}{2}}$ we get

$$
u^{-2 \epsilon}(y) G(y) \leq \frac{c}{(\epsilon R)^{2}}+\frac{c a}{\epsilon R}
$$

This proves that

$$
\begin{aligned}
\sup _{B_{p}(R)}\left(u^{2 \epsilon}|\nabla \log u|^{2}\right) & =\sup _{B_{p}(R)} \sigma \leq \sup _{B_{p}(2 R)} G \\
& \leq\left(\frac{c}{(\epsilon R)^{2}}+\frac{c a}{\epsilon R}\right) \sup _{B_{p}(2 R)}\left(u^{2 \epsilon}\right)
\end{aligned}
$$

We now observe that if $u$ is globally bounded on $M$, then the estimate implies $u$ is constant by letting $R \rightarrow \infty$. That means the gradient estimate claimed in the Proposition is automatically true. For unbounded $u$, we let

$$
\epsilon:=\left(2+\sup _{B_{p}(2 R)} \log (u+1)\right)^{-1}>0 .
$$

Then,

$$
\sup _{B_{p}(2 R)}\left(u^{2 \epsilon}\right) \leq \mathrm{e}^{2} .
$$

So we obtain, for any $R>0$ and $r<R$, that

$$
\begin{aligned}
\sup _{B_{p}(r)}\left(u^{2 \epsilon}|\nabla \log u|^{2}\right) \leq & \left(\frac{c}{R} \sup _{B_{p}(2 R)} \log (u+1)\right)^{2} \\
& +\frac{c a}{R} \sup _{B_{p}(2 R)} \log (u+1)+\frac{c(a+1)}{R} .
\end{aligned}
$$

Since $u$ is unbounded, it is clear that $\epsilon \rightarrow 0$ as $R \rightarrow \infty$. Therefore, after letting $R \rightarrow \infty$ with $r$ fixed, we arrive at

$$
\sup _{B_{p}(r)}|\nabla \log u|^{2} \leq C(n)\left(\Omega(u)^{2}+a \Omega(u)\right)
$$

Since $r$ is arbitrary, this proves the proposition. 
Let us point out that it is possible to prove another version of Proposition 3.1 without any growth assumption on $f$. It takes the form of $\sup _{M}$ $|\nabla \log u|^{2} \leq C\left(\Omega(u)^{2}+\Omega(u)\right)$ for some constant $C$ depending on the Ricci curvature lower bound and $\sup |\nabla f|$ on $B_{p}(1)$. This is because we can use the Laplace comparison Theorem 3.1 of [31] instead, which does not require any assumption on $f$. It in particular says that a positive $f$-harmonic function of sub-exponential growth on a complete manifold with $\operatorname{Ric}_{f} \geq 0$ must be a constant.

In the next step, we will establish an upper bound estimate for $\Omega(u)$ defined in Proposition 3.1 by using Moser iteration argument. First, we will establish a local Sobolev inequality on $M$, following the arguments in $[3,13$, $14,28]$. Since it will be crucial to have explicit and accurate dependency of the constants appearing in the inequality in terms of the growth of $f$, we provide details of the proof here.

We now use Lemma 2.1 to prove a Neumann Poincaré inequality. For this, we follow Buser's proof, see [3] (also cf.[5, p. 354]). There is an alternate proof, see [29, p. 176], which first establishes a weaker version of Neumann Poincare and then uses a covering argument to prove the strong version. For the proof of Theorem 3.1, the weaker version of Neumann Poincaré inequality is in fact sufficient. Recall

$$
A(R):=\sup _{x \in B_{p}(3 R)}|f|(x) .
$$

In the following, we will suppress $R$ in $A(R)$ and simply call it $A$.

Lemma 3.1. Let $\left(M, g, e^{-f} d v\right)$ be a smooth metric measure space with $R i c_{f} \geq 0$. Then for any $x \in B_{p}(R)$ we have

$$
\int_{B_{x}(r)}\left|\varphi-\varphi_{B_{x}(r)}\right|^{2} \leq c_{1} e^{c_{2} A} \cdot r^{2} \int_{B_{x}(r)}|\nabla \varphi|^{2}
$$

for all $0<r<R$ and $\varphi \in C^{\infty}\left(B_{x}(r)\right)$, where $\varphi_{B_{x}(r)}:=V^{-1}\left(B_{x}(r)\right) \int_{B_{x}(r)} \varphi$. The constants $c_{1}$ and $c_{2}$ depend only on the dimension $n$.

Proof of Lemma 3.1. First, we show that Lemma 2.1 and the argument in [3] imply a lower bound on the isoperimetric constant. Let $\Gamma$ be a smooth hypersurface in $B_{x}(r)$ with $\bar{\Gamma}$ imbedded in $\overline{B_{x}(r)}$. Let $D_{1}$ and $D_{2}$ be disjoint open subsets in $B_{x}(r)$ such that $D_{1} \cup D_{2}=B_{x}(r) \backslash \Gamma$. We will show that

$$
\min \left\{V\left(D_{1}\right), V\left(D_{2}\right)\right\} \leq r c_{1} \mathrm{e}^{c_{2} A} A(\Gamma) .
$$


Then, by Cheeger's theorem, the inequality (3.4) gives the claimed Neumann Poincaré inequality.

We fix $D_{1}$ so that $V\left(D_{1} \cap B_{x}\left(\frac{r}{2}\right)\right) \leq \frac{1}{2} V\left(B_{x}\left(\frac{r}{2}\right)\right)$. For a fixed $\alpha \in(0,1)$ to be chosen later, consider first the case when $V\left(D_{1} \cap B_{x}\left(\frac{r}{2}\right)\right) \leq \alpha V\left(D_{1}\right)$. We denote by $C(x)$ the cut-locus of $x$. For $y \in D_{1} \backslash C(x)$, let $y^{*}$ be the first intersection point of $y x$, the unique minimizing geodesic from $y$ to $x$, with $\Gamma$. In the case $y x$ does not intersect $\Gamma$, we set $y^{*}=x$.

Define

$$
\begin{aligned}
& \mathcal{A}_{1}:=\left\{y \in D_{1} \backslash\left(C(x) \cup B_{x}\left(\frac{r}{2}\right)\right): \quad y^{*} \notin B_{x}\left(\frac{r}{4}\right)\right\} \\
& \mathcal{A}_{2}:=\left\{y \in D_{1} \backslash\left(C(x) \cup B_{x}\left(\frac{r}{2}\right)\right): \quad y^{*} \in B_{x}\left(\frac{r}{4}\right)\right\} \\
& \mathcal{A}_{3}:=\left(B_{x}\left(\frac{r}{2}\right) \backslash B_{x}\left(\frac{r}{4}\right)\right) \cap\left(\cup_{y \in \mathcal{A}_{2}} \operatorname{rod}(y)\right),
\end{aligned}
$$

where $\operatorname{rod}(y):=\left\{\exp _{x}(\tau \xi): \quad \frac{r}{4}<\tau<s\right\}$ for $y=\exp _{x}(s \xi)$ with $\xi \in S_{x} M$ and $0<s<r$.

From now on, we will use $c_{1}$ and $c_{2}$ to denote constants depending only on dimension $n$. By Lemma 2.1 we have $\frac{V\left(\mathcal{A}_{2}\right)}{V\left(\mathcal{A}_{3}\right)} \leq c_{1} \mathrm{e}^{c_{2} A}$. Note that $V\left(\mathcal{A}_{1}\right)+$ $V\left(\mathcal{A}_{2}\right)=V\left(D_{1} \backslash B_{x}\left(\frac{r}{2}\right)\right) \geq(1-\alpha) V\left(D_{1}\right)$ and $V\left(\mathcal{A}_{3}\right) \leq V\left(D_{1} \cap B_{x}\left(\frac{r}{2}\right)\right) \leq$ $\alpha V\left(D_{1}\right)$. Thus,

$$
\begin{aligned}
(1-\alpha) V\left(D_{1}\right) & \leq V\left(\mathcal{A}_{1}\right)+V\left(\mathcal{A}_{2}\right) \leq V\left(\mathcal{A}_{1}\right)+c_{1} \mathrm{e}^{c_{2} A} V\left(\mathcal{A}_{3}\right) \\
& \leq V\left(\mathcal{A}_{1}\right)+\alpha c_{1} \mathrm{e}^{c_{2} A} V\left(D_{1}\right),
\end{aligned}
$$

or

$$
V\left(\mathcal{A}_{1}\right) \geq\left(1-\alpha c_{1} \mathrm{e}^{c_{2} A}\right) V\left(D_{1}\right)
$$

By setting

$$
\alpha:=\frac{1}{2}\left(c_{1}\right)^{-1} \mathrm{e}^{-c_{2} A},
$$

we conclude $V\left(\mathcal{A}_{1}\right) \geq \frac{1}{2} V\left(D_{1}\right)$. Moreover, Lemma 2.1 implies that

$$
\frac{A(\Gamma)}{V\left(\mathcal{A}_{1}\right)} \geq\left(c_{3}\right)^{-1} \mathrm{e}^{-c_{4} A} r^{-1}
$$

where constants $c_{3}$ and $c_{4}$ depending only on $n$.

This, together with (3.5), proves the lemma in the case when $V\left(D_{1} \cap\right.$ $\left.B_{x}\left(\frac{r}{2}\right)\right) \leq \alpha V\left(D_{1}\right)$, where $\alpha$ is given by $(3.6)$. 
Now we assume that $V\left(D_{1} \cap B_{x}\left(\frac{r}{2}\right)\right) \geq \alpha V\left(D_{1}\right)$ and finish the proof of the lemma. We need the following general fact, see [3]. Set $W_{0}:=D_{1} \cap$ $B_{x}\left(\frac{r}{2}\right)$ and $W_{1}:=D_{2} \cap B_{x}\left(\frac{r}{2}\right)$ or vice versa $W_{0}:=D_{2} \cap B_{x}\left(\frac{r}{2}\right)$ and $W_{1}:=$ $D_{1} \cap B_{x}\left(\frac{r}{2}\right)$. Then, for at least one of the two choices of $\left\{W_{0}, W_{1}\right\}$, there exist a point $w_{0} \in W_{0}$ and a measurable set $\mathcal{W}_{1} \subset W_{1}$ so that $V\left(\mathcal{W}_{1}\right) \geq$ $\frac{1}{2} V\left(W_{1}\right)$. Moreover, for each $y \in \mathcal{W}_{1}$, the minimizing geodesic $y w_{0}$ from $y$ to $w_{0}$ intersects $\Gamma$ and the first intersection point $y^{*}$ satisfies $d\left(y, y^{*}\right) \leq$ $d\left(y^{*}, w_{0}\right)$.

Observe that $\alpha V\left(D_{1}\right) \leq V\left(D_{1} \cap B_{x}\left(\frac{r}{2}\right)\right) \leq \frac{1}{2} V\left(B_{x}\left(\frac{r}{2}\right)\right)$. Therefore, $\alpha V\left(D_{1}\right) \leq V\left(D_{2} \cap B_{x}\left(\frac{r}{2}\right)\right)$, too. So, regardless of how $\mathcal{W}_{1}$ is picked, we have $\alpha V\left(D_{1}\right) \leq 2 V\left(\mathcal{W}_{1}\right)$. Now, to establish the lemma, we need only to show $\frac{A(\Gamma)}{V\left(\mathcal{W}_{1}\right)} \geq\left(c_{3}\right)^{-1} \mathrm{e}^{-c_{4} A} r^{-1}$ for some $c_{3}$ and $c_{4}$ depending only on $n$. For this, we use polar coordinates at $w_{0}$. For $y \in \mathcal{W}_{1}$, write $y=\exp _{w_{0}}\left(t_{0} \xi\right)$. Let $y^{*}$ be the first intersecting point of $y w_{0}$ with $\Gamma$. Define $t_{1}$ by $y^{*}=\exp _{w_{0}}\left(t_{1} \xi\right)$. From the choice of $w_{0}$ and $\mathcal{W}_{1}$, we know $t_{1} \geq \frac{1}{2} t_{0}$. Let $t_{2}$ be the maximal $t$ so that $\exp _{w_{0}}(t \xi) \in \mathcal{W}_{1} \backslash C\left(w_{0}\right)$. Clearly, $t_{2} \leq 2 t_{1}$. From Lemma 2.1 we conclude that

$$
\begin{aligned}
\int_{t_{1}}^{t_{2}} J\left(w_{0}, t, \xi\right) d t & \leq\left(t_{2}-t_{1}\right) J\left(w_{0}, t_{1}, \xi\right) c_{1} \mathrm{e}^{c_{2} A} \\
& \leq r J\left(w_{0}, t_{1}, \xi\right) c_{1} \mathrm{e}^{c_{2} A} .
\end{aligned}
$$

The desired result then follows after integrating in $\xi$. This proves the lemma.

Combining Lemma 2.1, Lemma 3.1 and the argument in [14], we get a local Neumann Sobolev inequality of the following form.

Lemma 3.2. Let $\left(M, g, e^{-f} d v\right)$ be a smooth metric measure space with Ric $_{f} \geq 0$. Then there exist constants $\nu>2, c_{1}$ and $c_{2}$, all depending only on $n$ such that

$$
\left(\int_{B_{p}(R)}\left|\varphi-\varphi_{B_{p}(R)}\right|^{\frac{2 \nu}{\nu-2}}\right)^{\frac{\nu-2}{\nu}} \leq c_{1} e^{c_{2} A} \frac{R^{2}}{V\left(B_{p}(R)\right)^{\frac{2}{\nu}}} \int_{B_{p}(R)}|\nabla \varphi|^{2}
$$

for $\varphi \in C^{\infty}\left(B_{p}(R)\right)$, where $\varphi_{B_{p}(R)}:=V^{-1}\left(B_{p}(R)\right) \int_{B_{p}(R)} \varphi$.

Proof of Lemma 3.2. For $y \in B_{p}(R)$, let $\gamma(t)$ be a minimizing geodesic from $p$ to $y$ such that $\gamma(0)=p$ and $\gamma(L)=y$. Define $y_{0}:=p$ and $y_{i}:=\gamma\left(\sum_{j=1}^{i} \frac{R}{2^{j}}\right)$ for $1 \leq i \leq i_{0}$, where $i_{0}$ is the largest integer $i$ so that $\sum_{j=1}^{i} \frac{R}{2^{j}}<L$. Define also $B_{i}:=B_{y_{i}}\left(\frac{R}{2^{i+1}}\right)$ for $i<i_{0}$ and $B_{i}:=B_{y}\left(\frac{R}{2^{i+1}}\right)$ for $i \geq i_{0}$. 
Let $\varphi_{B_{i}}:=V^{-1}\left(B_{i}\right) \int_{B_{i}} \varphi$. Then $\lim _{i \rightarrow \infty} \varphi_{B_{i}}=\varphi(y)$. Thus, we have

$$
\left|\varphi_{B_{0}}-\varphi(y)\right| \leq \sum_{i \geq 0}\left|\varphi_{B_{i}}-\varphi_{B_{i+1}}\right| \leq \sum_{i \geq 0}\left(\left|\varphi_{B_{i}}-\varphi_{D_{i}}\right|+\left|\varphi_{D_{i}}-\varphi_{B_{i+1}}\right|\right) .
$$

Here, $D_{i}:=B_{z_{i}}\left(\frac{R}{2^{i+3}}\right) \subset B_{i} \cap B_{i+1}$, and $z_{i}:=\gamma\left(\sum_{j=1}^{i} \frac{R}{2^{j}}+\frac{3 R}{2^{i+3}}\right)$.

By Lemma 2.1 it is easy to see that

$$
\left|\varphi_{B_{i}}-\varphi_{D_{i}}\right| \leq V\left(D_{i}\right)^{-1} \int_{B_{i}}\left|\varphi-\varphi_{B_{i}}\right| \leq c_{1} \mathrm{e}^{c_{2} A} V\left(B_{i}\right)^{-1} \int_{B_{i}}\left|\varphi-\varphi_{B_{i}}\right| .
$$

Note that a similar bound for $\left|\varphi_{D_{i}}-\varphi_{B_{i+1}}\right|$ also holds. So we conclude that

$$
\begin{aligned}
\left|\varphi_{B_{0}}-\varphi(y)\right| & \leq c_{1} \mathrm{e}^{c_{2} A} \sum_{i \geq 0} V\left(B_{i}\right)^{-1} \int_{B_{i}}\left|\varphi-\varphi_{B_{i}}\right| \\
& \leq c_{1} \mathrm{e}^{c_{2} A} \sum_{i \geq 0}\left(V\left(B_{i}\right)^{-1} \int_{B_{i}}\left|\varphi-\varphi_{B_{i}}\right|^{2}\right)^{\frac{1}{2}} \\
& \leq c_{1} \mathrm{e}^{c_{2} A} \sum_{i \geq 0} \frac{R}{2^{i+1}}\left(V\left(B_{i}\right)^{-1} \int_{B_{i}}|\nabla \varphi|^{2}\right)^{\frac{1}{2}}
\end{aligned}
$$

where in the second line we have use the Cauchy-Schwarz inequality and in the last line we have used Lemma 3.1. On the other hand,

$$
\left|\varphi_{B_{0}}-\varphi(y)\right|=c R^{-\frac{1}{2}} \sum_{i \geq 0}\left(\frac{R}{2^{i+1}}\right)^{\frac{1}{2}}\left|\varphi_{B_{0}}-\varphi(y)\right|,
$$

where $c$ is a universal constant. So for $R_{i}:=\frac{R}{2^{i+1}}$, we have

$$
\sum_{i \geq 0}\left(R_{i}\right)^{\frac{1}{2}}\left|\varphi_{B_{0}}-\varphi(y)\right| \leq c_{1} \mathrm{e}^{c_{2} A} R^{\frac{1}{2}} \sum_{i \geq 0} R_{i}\left(V\left(B_{i}\right)^{-1} \int_{B_{i}}|\nabla \varphi|^{2}\right)^{\frac{1}{2}} .
$$

Hence, there exists an $i$ (depending on $y$ ) so that

$$
\left|\varphi_{B_{0}}-\varphi(y)\right|^{2} \leq c_{1} \mathrm{e}^{c_{2} A}\left(R R_{i}\right) \frac{1}{V\left(B_{i}\right)} \int_{B_{i}}|\nabla \varphi|^{2}
$$

Since $B_{i} \subset B_{y}\left(3 R_{i}\right)$, it follows that for each $y \in B_{p}(R)$ there exists $r_{y}>0$ so that

$$
\left|\varphi_{B_{0}}-\varphi(y)\right|^{2} \leq c_{1} \mathrm{e}^{c_{2} A}\left(R r_{y}\right) V\left(B_{y}\left(r_{y}\right)\right)^{-1} \int_{B_{y}\left(r_{y}\right) \cap B_{p}(R)}|\nabla \varphi|^{2}
$$


According to Lemma 2.1,

$$
\frac{V\left(B_{p}(R)\right)}{V\left(B_{y}\left(r_{y}\right)\right)} \leq \frac{V\left(B_{y}(2 R)\right)}{V\left(B_{y}\left(r_{y}\right)\right)} \leq c_{1} \mathrm{e}^{c_{2} A}\left(\frac{R}{r_{y}}\right)^{n}
$$

Solving $r_{y}$ from (3.8) and plugging into (3.7) then gives

$$
\begin{aligned}
\left|\varphi_{B_{0}}-\varphi(y)\right|^{2} \leq & c_{1} \mathrm{e}^{c_{2} A} R^{2} V\left(B_{p}(R)\right)^{-\frac{1}{n}} V\left(B_{y}\left(r_{y}\right)\right)^{\frac{1}{n}-1} \\
& \times \int_{B_{y}\left(r_{y}\right) \cap B_{p}(R)}|\nabla \varphi|^{2}
\end{aligned}
$$

We now define $A_{t}:=\left\{y \in B_{p}(R):\left|\varphi_{B_{0}}-\varphi(y)\right| \geq t\right\}$. Applying the Vitali covering lemma, we find a countable disjoint collection $\left\{B_{i}\left(r_{i}\right)\right\}_{i \in I}$ of balls from $\left\{B_{y}\left(r_{y}\right): y \in A_{t}\right\}$ such that for any $y \in A_{t}$, there exists $i \in I$ such that $B_{i}\left(r_{i}\right) \cap B_{y}\left(r_{y}\right) \neq \phi$ and $B_{y}\left(r_{y}\right) \subset B_{i}\left(3 r_{i}\right)$. Then it follows, by Lemma 2.1 and (3.9), that

$$
\begin{aligned}
V\left(A_{t}\right)^{1-\frac{1}{n}} & \leq c_{1} \mathrm{e}^{c_{2} A} \sum_{i \in I} V\left(B_{i}\right)^{1-\frac{1}{n}} \\
& \leq c_{1} \mathrm{e}^{c_{2} A} \frac{R^{2}}{t^{2}} V\left(B_{p}(R)\right)^{-\frac{1}{n}} \sum_{i \in I} \int_{B_{i}\left(r_{i}\right) \cap B_{p}(R)}|\nabla \varphi|^{2} \\
& =c_{1} \mathrm{e}^{c_{2} A} \frac{R^{2}}{t^{2}} V\left(B_{p}(R)\right)^{-\frac{1}{n}} \int_{B_{p}(R)}|\nabla \varphi|^{2} .
\end{aligned}
$$

This may be rewritten as

$$
V\left(A_{t}\right) \leq t^{-\frac{2 n}{n-1}} B
$$

with $B:=c_{1} \mathrm{e}^{c_{2} A} R^{\frac{2 n}{n-1}} V\left(B_{p}(R)\right)^{-\frac{1}{n-1}}\left(\int_{B_{p}(R)}|\nabla \varphi|^{2}\right)^{\frac{n}{n-1}}$. Now, for any $\frac{2 n}{n-1}>q>2$, we have

$$
\begin{aligned}
\int_{B_{p}(R)}\left|\varphi-\varphi_{B_{0}}\right|^{q} & =q \int_{0}^{\infty} t^{q-1} V\left(A_{t}\right) d t \\
& =q \int_{0}^{T} t^{q-1} V\left(A_{t}\right) d t+q \int_{T}^{\infty} t^{q-1} V\left(A_{t}\right) d t \\
& \leq T^{q} V\left(B_{p}(R)\right)+\frac{q}{\frac{2 n}{n-1}-q} T^{q-\frac{2 n}{n-1}} B
\end{aligned}
$$


where we have used (3.10) to bound the second integral in the second line. Choosing $q=\frac{2 n-1}{n-1}$ and $T:=\left(R^{2} \frac{1}{V\left(B_{p}(R)\right)} \int_{B_{p}(R)}|\nabla \varphi|^{2}\right)^{\frac{1}{2}}$, we get

$$
\left(\int_{B_{p}(R)}\left|\varphi-\varphi_{B_{0}}\right|^{\frac{2 \nu}{\nu-2}}\right)^{\frac{\nu-2}{\nu}} \leq c_{1} \mathrm{e}^{c_{2} A} \frac{R^{2}}{V\left(B_{p}(R)\right)^{\frac{2}{\nu}}} \int_{B_{p}(R)}|\nabla \varphi|^{2},
$$

where $\nu:=\frac{2 q}{q-2}=4 n-2$. This proves the theorem.

We are now ready to prove Theorem 3.1.

Proof of Theorem 3.1. Let $u$ be a positive solution to $\Delta_{f} u=0$. Applying the Moser iteration scheme to the equation $\Delta_{f} u=0$ with the help of Lemma 2.1 and Lemma 3.2, we obtain

$$
\sup _{B_{p}\left(\frac{1}{2} R\right)} u \leq \frac{c_{1} \mathrm{e}^{c_{2} A}}{V\left(B_{p}(R)\right)} \int_{B_{p}(R)} u,
$$

where $c_{1}$ and $c_{2}$ are constants depending only on $n$. For this, notice that the Neumann Sobolev inequality, Lemma 3.2, also holds true when integrals are with respect to the measure $\mathrm{e}^{-f} d v$, without changing the nature of the dependency of the constants on $A$.

Now we start using the assumption on $f$ that $|f|(x) \leq a r(x)+b$ on $M$. The following argument follows closely the proof of Proposition 2.1 in [30]. Applying (2.3) we obtain, for any $r>0$, that $\Delta_{f} r(x) \leq \frac{n-1+4 b}{r}+3 a$. Therefore, we can find $r_{0}>0$ so that

$$
\Delta_{f} r(x) \leq 4 a \text { for any } x \in M \backslash B_{p}\left(r_{0}\right) .
$$

So for $r>r_{0}$,

$$
\begin{aligned}
4 a \int_{B_{p}(r) \backslash B_{p}\left(r_{0}\right)} u \mathrm{e}^{-f} \geq & \int_{B_{p}(r) \backslash B_{p}\left(r_{0}\right)} u\left(\Delta_{f} r\right) \mathrm{e}^{-f} \\
= & r_{0} \int_{\partial B_{p}\left(r_{0}\right)}\langle\nabla u, \nabla r\rangle \mathrm{e}^{-f}-r \int_{\partial B_{p}(r)}\langle\nabla u, \nabla r\rangle \mathrm{e}^{-f} \\
& +\int_{\partial B_{p}(r)} u \mathrm{e}^{-f}-\int_{\partial B_{p}\left(r_{0}\right)} u \mathrm{e}^{-f} \\
= & \int_{\partial B_{p}(r)} u \mathrm{e}^{-f}-\int_{\partial B_{p}\left(r_{0}\right)} u \mathrm{e}^{-f},
\end{aligned}
$$


where we have used the fact that $\Delta_{f} u=0$ and

$$
\int_{\partial B_{p}(r)}\langle\nabla u, \nabla r\rangle \mathrm{e}^{-f}=\int_{B_{p}(r)}\left(\Delta_{f} u\right) \mathrm{e}^{-f}=0 .
$$

Denote by

$$
\begin{aligned}
U(r) & :=\int_{B_{p}(r) \backslash B_{p}\left(r_{0}\right)} u \mathrm{e}^{-f} \text { and } \\
C_{0} & :=\int_{\partial B_{p}\left(r_{0}\right)} u \mathrm{e}^{-f} .
\end{aligned}
$$

Then the preceding inequality implies that for $r>r_{0}$,

$$
U^{\prime}(r) \leq 4 a U(r)+C_{0}
$$

After integrating from $r_{0}$ to $R>r_{0}$, we obtain

$$
U(R) \leq C_{1} \mathrm{e}^{4 a R}
$$

with $C_{1}:=U\left(r_{0}\right)+\frac{1}{a} C_{0}$. Consequently, we have

$$
\int_{B_{p}(R)} u \leq C_{2} \mathrm{e}^{5 a R}
$$

Plugging this into (3.11), we conclude that

$$
\sup _{B_{p}\left(\frac{1}{2} R\right)} u \leq C_{3} \mathrm{e}^{c(n) a R},
$$

where $c(n)$ depends only on dimension $n$ and $C_{3}$ is independent of $R$. This shows that

$$
\Omega(u):=\limsup _{R \rightarrow \infty}\left\{\frac{1}{R} \sup _{B_{p}(R)} \log (u+1)\right\} \leq c(n) a,
$$

where $c(n)$ depends only on $n$. By Proposition 3.1, we conclude

$$
\sup _{M}|\nabla \log u| \leq C(n) a
$$

with $C(n)$ being a constant depending only on $n$, as claimed in Theorem 3.1. It is evident that if $f$ has sublinear growth, then $a$ can be taken as close to zero as we wish, thus proving that $u$ must be constant. 
We now turn to polynomial growth $f$-harmonic functions.

Theorem 3.2. Let $\left(M, g, e^{-f} d v\right)$ be a complete non-compact smooth metric measure space with Ric $_{f} \geq 0$ and $f$ bounded. Then a sublinear growth $f$-harmonic function on $M$ must be a constant.

Proof of Theorem 3.2. Let $w$ be an $f$-harmonic function so that

$$
\lim _{x \rightarrow \infty} \frac{|w|(x)}{r(x)}=0
$$

The Bochner formula asserts that

$$
\frac{1}{2} \Delta_{f}|\nabla w|^{2}=\left|w_{i j}\right|^{2}+\operatorname{Ric}_{f}(\nabla w, \nabla w) \geq 0
$$

So $|\nabla w|^{2}$ is $f$-subharmonic. Applying the Moser iteration scheme, we obtain a mean value inequality of the form

$$
\sup _{B_{p}\left(\frac{1}{2} R\right)}|\nabla w|^{2} \leq \frac{C}{V\left(B_{p}(R)\right)} \int_{B_{p}(R)}|\nabla w|^{2} \mathrm{e}^{-f}
$$

for some constant $C$ depending on $n$ and sup $|f|$. Note that now the constant $A$ in Lemma 2.1 and Lemma 3.2 is independent of $R$ as $f$ is assumed to be bounded.

We now choose a cut-off $\phi$ such that $\phi=1$ on $B_{p}(R), \phi=0$ on $M \backslash B_{p}$ $(2 R)$ and $|\nabla \phi| \leq \frac{C}{R}$. Integrating by parts and using $\Delta_{f} w=0$ we get

$$
\begin{aligned}
\int_{M}|\nabla w|^{2} \phi^{2} \mathrm{e}^{-f} & =-2 \int_{M} w \phi\langle\nabla w, \nabla \phi\rangle \mathrm{e}^{-f} \\
& \leq 2 \int_{M}|w| \phi|\langle\nabla w, \nabla \phi\rangle| \mathrm{e}^{-f} \\
& \leq \frac{1}{2} \int_{M}|\nabla w|^{2} \phi^{2} \mathrm{e}^{-f}+2 \int_{M} w^{2}|\nabla \phi|^{2} \mathrm{e}^{-f}
\end{aligned}
$$


This shows that

$$
\begin{aligned}
\int_{B_{p}(R)}|\nabla w|^{2} \mathrm{e}^{-f} & \leq 4 \int_{M} w^{2}|\nabla \phi|^{2} \mathrm{e}^{-f} \leq \frac{C}{R^{2}} \int_{B_{p}(2 R) \backslash B_{p}(R)} w^{2} \mathrm{e}^{-f} \\
& \leq \frac{C}{R^{2}}\left(\sup _{B_{p}(2 R)} w^{2}\right) V\left(B_{p}(2 R)\right) \\
& \leq \frac{C}{R^{2}}\left(\sup _{B_{p}(2 R)} w^{2}\right) V\left(B_{p}(R)\right)
\end{aligned}
$$

where in the last line we have used Lemma 2.1.

Together with (3.13) we obtain

$$
\lim _{R \rightarrow \infty} \frac{1}{V\left(B_{p}(R)\right)} \int_{B_{p}(R)}|\nabla w|^{2} \mathrm{e}^{-f}=0 .
$$

So by (3.14), $|\nabla w|=0$ on $M$. The theorem is proved.

Our next result is a dimension estimate for the space of polynomial growth $f$-harmonic functions. For this, we use again Moser iteration and Sobolev inequality. However, the situation here is considerably easier as $f$ is assumed to be bounded.

Theorem 3.3. Let $\left(M, g, e^{-f} d v\right)$ be a complete non-compact smooth metric measure space with Ric $_{f} \geq 0$ and $f$ bounded. We have

$$
\operatorname{dim} \mathcal{H}^{d}(M) \leq C d^{n-1} \text { for any } d \geq 1
$$

Moreover, we have the sharp estimate

$$
\operatorname{dim} \mathcal{H}^{1}(M) \leq n+1
$$

Proof of Theorem 3.3. We first establish the second result about the dimension of the space of linear growth $f$-harmonic functions. Consider $u$ an $f$-harmonic function with linear growth, i.e., $\Delta_{f} u=0$ and $|u|(x) \leq C$ $(r(x)+1)$ on $M$. Using the argument in the proof of Theorem 3.2 we want to claim that $|\nabla u|$ is bounded on $M$. Indeed, since $f$ is bounded, (3.14) is true here, too. Now the reverse Poincaré inequality for $f$-harmonic function yields

$$
\frac{1}{V\left(B_{p}(R)\right)} \int_{B_{p}(R)}|\nabla u|^{2} \mathrm{e}^{-f} \leq \frac{C}{R^{2}} \sup _{B_{p}(2 R)} u^{2} \leq C .
$$

By (3.14) this shows that $|\nabla u|$ is bounded on $M$. 
We now prove a mean value theorem at infinity of the following form. For any bounded positive $f$-subharmonic function $v$ we have

$$
\lim _{R \rightarrow \infty} \frac{1}{V_{f}\left(B_{p}(R)\right)} \int_{B_{p}(R)} v \mathrm{e}^{-f}=\sup _{M} v
$$

When $f$ is constant this was first established by P. Li [36] by a heat equation method. Here, we follow the argument in [6] which uses a monotonicity formula.

Let $w=\sup _{M} v-v$, which is a positive function that satisfies

$$
\Delta_{f} w \leq 0 \text { and } \quad \inf _{M} w=0
$$

To prove (3.15) we show instead

$$
\lim _{R \rightarrow \infty} \frac{1}{V_{f}\left(B_{p}(R)\right)} \int_{B_{p}(R)} w \mathrm{e}^{-f}=0 .
$$

Let $h_{R}$ solve $\Delta_{f} h_{R}=0$ in $B_{p}(R)$ with $h_{R}=w$ on $\partial B_{p}(R)$. By the maximum principle, $h_{R}$ is positive and uniformly bounded. Moreover, since $\inf _{M} w=0$, for any $\varepsilon>0$ there exists $R_{\varepsilon}>0$ such that

$$
\inf _{B_{p}(R)} w<\varepsilon \quad \text { for any } R>R_{\varepsilon} .
$$

Again, by the maximum principle, it follows that

$$
\inf _{B_{p}(R)} h_{R}<\varepsilon \quad \text { for any } R>R_{\varepsilon} \text {. }
$$

Notice the following Harnack inequality holds.

$$
\sup _{B_{p}\left(\frac{1}{2} R\right)} h_{R} \leq C \inf _{B_{p}\left(\frac{1}{2} R\right)} h_{R}
$$

where $C$ depends only on $n$ and $\sup _{M}|f|$. Indeed, this follows from Lemma 2.1, Lemma 3.1 and Lemma 3.2 by the Moser iteration argument as in $[29$, Chapter II]. 
We therefore conclude that

$$
\sup _{B_{p}\left(\frac{1}{2} R\right)} h_{R}<C \varepsilon \quad \text { for any } R>2 R_{\varepsilon} \text {. }
$$

Furthermore, for $R>r>0$, we have

$$
\begin{aligned}
0 & =\int_{B_{p}(r)}\left(\Delta_{f} h_{R}\right) \mathrm{e}^{-f}=\int_{\partial B_{p}(r)} \frac{\partial h_{R}}{\partial r} \mathrm{e}^{-f} \\
& =\frac{\partial}{\partial r} \int_{\partial B_{p}(r)} h_{R} \mathrm{e}^{-f}-\int_{\partial B_{p}(r)} h_{R} \Delta_{f}(r) \mathrm{e}^{-f} \\
& \geq \frac{\partial}{\partial r} \int_{\partial B_{p}(r)} h_{R} \mathrm{e}^{-f}-\frac{C}{r} \int_{\partial B_{p}(r)} h_{R} \mathrm{e}^{-f},
\end{aligned}
$$

where in the last line we have used (2.3) and the fact that $f$ is bounded. This shows that $\log \left(\frac{1}{r^{C}} \int_{\partial B_{p}(r)} h_{R} \mathrm{e}^{-f}\right)$ is decreasing as a function of $r$ for $0<r<R$. In particular, it shows that

$$
\int_{\partial B_{p}(R)} h_{R} \mathrm{e}^{-f} \leq C \int_{\partial B_{p}\left(\frac{1}{2} R\right)} h_{R} \mathrm{e}^{-f}
$$

for a constant $C$ depending only on $n$ and $\sup _{M}|f|$.

So for $R>2 R_{\varepsilon}$,

$$
\begin{aligned}
\int_{\partial B_{p}(R)} w \mathrm{e}^{-f} & =\int_{\partial B_{p}(R)} h_{R} \mathrm{e}^{-f} \leq C \int_{\partial B_{p}\left(\frac{1}{2} R\right)} h_{R} \mathrm{e}^{-f} \\
& \leq C \varepsilon A_{f}\left(\partial B_{p}\left(\frac{1}{2} R\right)\right)
\end{aligned}
$$

where in the second line we have used (3.17). Since this inequality is true for all $R>2 R_{\varepsilon}$, integrating this from $2 R_{\varepsilon}$ to $R$ gives

$$
\begin{aligned}
\int_{B_{p}(R) \backslash B_{p}\left(2 R_{\varepsilon}\right)} w \mathrm{e}^{-f} & \leq C \varepsilon \int_{2 R_{\varepsilon}}^{R} A_{f}\left(\partial B_{p}\left(\frac{1}{2} t\right)\right) d t \\
& \leq C \varepsilon V_{f}\left(B_{p}\left(\frac{1}{2} R\right)\right) .
\end{aligned}
$$


Hence, for $R$ sufficiently large,

$$
\begin{aligned}
\frac{1}{V_{f}\left(B_{p}(R)\right)} \int_{B_{p}(R)} w \mathrm{e}^{-f}= & \frac{1}{V_{f}\left(B_{p}(R)\right)} \int_{B_{p}(R) \backslash B_{p}\left(2 R_{\varepsilon}\right)} w \mathrm{e}^{-f} \\
& +\frac{1}{V_{f}\left(B_{p}(R)\right)} \int_{B_{p}\left(2 R_{\varepsilon}\right)} w \mathrm{e}^{-f} \\
\leq & C \varepsilon+\frac{1}{V_{f}\left(B_{p}(R)\right)} \int_{B_{p}\left(2 R_{\varepsilon}\right)} w \mathrm{e}^{-f} \leq 2 C \varepsilon
\end{aligned}
$$

This proves (3.16).

The rest of the argument now follows verbatim [18]. For completeness we sketch it below.

For $u, v \in \mathcal{H}^{1}(M)$ define

$$
\langle\langle u, v\rangle\rangle:=\lim _{R \rightarrow \infty} \frac{1}{V_{f}\left(B_{p}(R)\right)} \int_{B_{p}(R)}\langle\nabla u, \nabla v\rangle \mathrm{e}^{-f} .
$$

This is well defined in view of (3.16). Also, $\langle\langle\rangle$,$\rangle defines an inner product on$

$$
\mathcal{H}^{\prime}:=\left\{u \in \mathcal{H}^{1}(M): u(p)=0\right\}
$$

Consider any finite dimensional subspace $\mathcal{H}^{\prime \prime}$ of $\mathcal{H}^{\prime}$, of dimension $l$. Let $\left\{u_{1}, \ldots, u_{l}\right\}$ be an orthonormal basis of $\left(\mathcal{H}^{\prime \prime},\langle\langle\rangle\rangle,\right)$ and define

$$
F^{2}(x):=\sum_{i=1}^{l} u_{i}^{2}(x)
$$

Note $F$ is independent of the choice of $\left\{u_{i}\right\}$. For a fixed point $x \in M$, we may choose $\left\{u_{i}\right\}$ so that $u_{i}(x)=0$ for all $i \neq 1$. Then it follows that

$$
\begin{aligned}
F^{2}(x) & =u_{1}^{2}(x) \quad \text { and } \\
F(x) \nabla F(x) & =u_{1}(x) \nabla u_{1}(x) .
\end{aligned}
$$

Since $\left\langle\left\langle u_{1}, u_{1}\right\rangle\right\rangle=1$, we have

$$
\sup _{M}\left|\nabla u_{1}\right|=1
$$


This shows that $|\nabla F|(x) \leq 1$, too. Integrating along minimizing geodesics and using $F(p)=0$, we get that $F(x) \leq r(x)$. On the other hand,

$$
\begin{aligned}
2 \sum_{i=1}^{l} \int_{B_{p}(R)}\left|\nabla u_{i}\right|^{2} \mathrm{e}^{-f} & =\int_{B_{p}(R)}\left(\Delta_{f} F^{2}\right) \mathrm{e}^{-f} \\
& \leq 2 \int_{\partial B_{p}(R)} F|\nabla F| \mathrm{e}^{-f} \leq 2 R A_{f}\left(\partial B_{p}(R)\right) .
\end{aligned}
$$

Since $\left\{u_{i}\right\}$ is orthonormal with respect to $\langle\langle\rangle$,$\rangle , for any \varepsilon>0$ there exists $R_{\varepsilon}$ such that for $R>R_{\varepsilon}$,

$$
\sum_{i=1}^{l} \frac{1}{V_{f}\left(B_{p}(R)\right)} \int_{B_{p}(R)}\left|\nabla u_{i}\right|^{2} \mathrm{e}^{-f} \geq l-\varepsilon
$$

So, according to (3.18), for any $R \geq R_{\varepsilon}$,

$$
\frac{l-\varepsilon}{R} \leq \frac{\left(V_{f}\left(B_{p}(R)\right)\right)^{\prime}}{V_{f}\left(B_{p}(R)\right)} .
$$

Integrating the inequality from $R_{\varepsilon}$ to $R$, we then conclude that

$$
\left(\frac{R}{R_{\varepsilon}}\right)^{l-\varepsilon} \leq \frac{V_{f}\left(B_{p}(R)\right)}{V_{f}\left(B_{p}\left(R_{\varepsilon}\right)\right)}
$$

On the other hand, according to Lemma 2.1, we have

$$
V_{f}\left(B_{p}(R)\right) \leq C R^{n}
$$

Plugging into (3.19), we conclude that

$$
\operatorname{dim} \mathcal{H}^{1}(M) \leq n+1
$$

This proves the second claim of Theorem 3.3.

To prove that $\operatorname{dim} \mathcal{H}^{d}(M) \leq C d^{n-1}$ for $d>1$, we observe first that since $f$ is bounded we have for any $x \in M$,

$$
\frac{V\left(B_{x}(R)\right)}{V\left(B_{x}(r)\right)} \leq C_{v}\left(\frac{R}{r}\right)^{n} \quad \text { for } r<R,
$$


where $C_{v}$ depends on $n$ and $\sup _{M}|f|$. Moreover, the Sobolev inequality Lemma 3.2 and the Moser iteration imply that we have a mean value inequality of the form

$$
u^{2}(x) \leq C_{\mathcal{M}} \frac{1}{V\left(B_{x}(R)\right)} \int_{B_{x}(R)} u^{2}
$$

for any non-negative $f$-subharmonic function $u$ on $M$. The result in [17] then implies $\operatorname{dim} \mathcal{H}^{d}(M) \leq C d^{n-1}$. The theorem is proved.

\section{Rigidity}

In this section, we investigate the structure at infinity of smooth metric measure spaces whose $\lambda_{1}\left(\Delta_{f}\right)$ attains its upper bound in Theorem 2.1. We prove our result by using a Busemann function argument, which is similar to the one in [21]. A manifold $M$ is called connected at infinity if it has only one end.

Theorem 4.1. Let $\left(M, g, e^{-f} d v\right)$ be a smooth metric measure space such that Ric R $_{f} \geq 0$. Assume that $\lambda_{1}\left(\Delta_{f}\right)=\frac{1}{4} a^{2}$, where $a$ is the linear growth rate of $f$. Then, either $M$ is connected at infinity or $M$ is isometric to $\mathbb{R} \times N$ for some compact manifold $N$.

Before proving the theorem, we recall some terminology. First, a manifold is $f$-non-parabolic if $\Delta_{f}$ admits a positive Green's function. Otherwise, it is called $f$-parabolic. For an end of the manifold, the same definition applies, where the Green's function now refers to the one satisfying the Neumann boundary conditions. We will divide our proof into two cases according to the type of ends of $M$. In fact, the result for $f$-non-parabolic ends does not require any assumption on $f$ or $\lambda_{1}\left(\Delta_{f}\right)$. We state it in the following lemma.

Lemma 4.1. Let $\left(M, g, e^{-f} d v\right)$ be a smooth metric measure space with $R i c_{f} \geq 0$. Then $M$ has at most one $f$-non-parabolic end.

Proof of Lemma 4.1. Suppose $M$ has two $f$-non-parabolic ends. Then $M$ admits a positive non-constant bounded $f$-harmonic function $v$ with $\int_{M}|\nabla v|^{2}$ $\mathrm{e}^{-f}<\infty$. This kind of result was first discovered by Li and Tam (see [35]). Now according to a result of Brighton [2], since $v$ is bounded, it must be a constant function. This is a contradiction. 
Alternatively, the lemma may be proved as follows. Using the Bochner formula

$$
\frac{1}{2} \Delta_{f}|\nabla v|^{2}=\left|v_{i j}\right|^{2}+\left\langle\nabla \Delta_{f} v, \nabla v\right\rangle+\operatorname{Ric}_{f}(\nabla v, \nabla v) \geq\left|v_{i j}\right|^{2}
$$

and a cut-off argument, we get

$$
\begin{aligned}
& 2 \int_{M}\left|v_{i j}\right|^{2} \mathrm{e}^{-f} \phi^{2} \leq \int_{M}\left(\Delta_{f}|\nabla v|^{2}\right) \phi^{2} \mathrm{e}^{-f} \\
& \quad=-\int_{M}\left\langle\nabla|\nabla v|^{2}, \nabla \phi^{2}\right\rangle \mathrm{e}^{-f} \\
& \quad \leq \int_{M}\left|v_{i j}\right|^{2} \phi^{2} \mathrm{e}^{-f}+4 \int_{M}|\nabla v|^{2}|\nabla \phi|^{2} \mathrm{e}^{-f} .
\end{aligned}
$$

Thus,

$$
\int_{M}\left|v_{i j}\right|^{2} \mathrm{e}^{-f} \phi^{2} \leq 4 \int_{M}|\nabla v|^{2}|\nabla \phi|^{2} \mathrm{e}^{-f} .
$$

Since $\int_{M}|\nabla v|^{2} \mathrm{e}^{-f}<\infty$, the right hand side goes to 0 after taking the limit $R \rightarrow \infty$ by choosing $\phi$ to be the standard cut-off function so that $\phi=1$ on $B_{p}(R)$ and $\phi=0$ outside $B_{p}(2 R)$. This forces $v_{i j}=0$. Therefore, $|\nabla v|$ must be a constant on $M$. Now the weighted volume $V_{f}(M)=\int_{M} \mathrm{e}^{-f} d v=\infty$ as $M$ is $f$-non-parabolic. Using $\int_{M}|\nabla v|^{2} \mathrm{e}^{-f}<\infty$ again, we conclude $|\nabla v|=0$ and $v$ is a constant. This finishes our proof.

We now prove Theorem 4.1.

Proof of Theorem 4.1. First, since $\lambda_{1}\left(\Delta_{f}\right)>0$, we know that $M$ is $f$-nonparabolic. This follows exactly as in the case $f=$ constant. We refer to [16] for details.

Let us assume that $M$ has at least two ends. By Lemma 4.1 exactly one end is $f$-non-parabolic, and all other are $f$-parabolic. We denote $E$ to be the $f$-non-parabolic end and let $F:=M \backslash E$. Then $F$ is an $f$-parabolic end.

First, we claim the fact that $\lambda_{1}\left(\Delta_{f}\right)=\frac{1}{4} a^{2}$ implies

$$
V_{f}\left(F \backslash B_{p}(R)\right) \leq C \mathrm{e}^{-a R} .
$$

This claim can be verified by following the argument of Li and Wang in [20]. Since they only use the variational principle for the bottom spectrum and integration by parts, it is easy to check that their estimates can be carried over to our setting. We shall omit the details here. 
For a geodesic ray $\gamma$ contained in the end $F$, define the associated Busemann function by

$$
\beta(x):=\lim _{t \rightarrow \infty}(t-d(x, \gamma(t))) .
$$

Denote by $\tau_{t}(s)$ the normal minimizing geodesic from $\gamma(t)$ to $x$. According to $(2.3)$ we have

$$
\Delta_{f}(d(\gamma(t), x)) \leq \frac{n-1}{r}-\frac{2}{r} f(x)+\frac{2}{r^{2}} \int_{0}^{r} f(s) d s
$$

where $r:=d(\gamma(t), x)$ and $f(s):=f\left(\tau_{t}(s)\right)$. Let us assume that there exist $\alpha>0$ and $C>0$ such that $|f(z)| \leq \alpha d(p, z)+C$, for all $z \in M$. Then,

$$
\begin{aligned}
|f(s)| & =\left|f\left(\tau_{t}(s)\right)\right| \leq \alpha d\left(p, \tau_{t}(s)\right)+C \\
& \leq \alpha\left(d(p, x)+d\left(x, \tau_{t}(s)\right)\right)+C \\
& =\alpha(r-s)+(\alpha d(p, x)+C),
\end{aligned}
$$

for any $0<s<r$. Therefore, since $|f(x)| \leq \alpha d(p, x)+C$, we conclude

$$
\begin{aligned}
\Delta_{f}(d(\gamma(t), x)) \leq & \frac{n-1+4(\alpha d(p, x)+C)}{r}+\frac{2}{r^{2}} \int_{0}^{r} \alpha(r-s) d s \\
& =\frac{n-1+4(\alpha d(p, x)+C)}{r}+\alpha .
\end{aligned}
$$

Now, by using this and the definition of the Busemann function, it is standard to see that the following inequality holds in the sense of distributions:

$$
\Delta_{f} \beta(x) \geq-\alpha
$$

Now, if $a$ denotes the linear growth rate of $f$ i.e., it is the infimum of such $\alpha$, it follows that

$$
\Delta_{f} \beta(x) \geq-a
$$

To estimate the volume growth of the $f$-non-parabolic end $E$ we will use (4.2) and the fact that $|\nabla \beta|=1$. Note

$$
\Delta_{f} e^{a \beta} \geq 0
$$

We also know that on the end $E$ the Busemann function is equivalent to the distance function, that is, there exists a constant $C$ such that

$$
-r(x)-C \leq \beta(x) \leq-r(x)+C
$$


for $x \in E$, see [21]. Integrating $\Delta_{f} \mathrm{e}^{a \beta}$ on $\{-t \leq \beta \leq-r\} \cap E$ and using Stokes theorem we get

$$
\begin{aligned}
0 & \leq \frac{1}{a} \int_{\{-t \leq \beta \leq-r\} \cap E}\left(\Delta_{f} \mathrm{e}^{a \beta}\right) \mathrm{e}^{-f} \\
& =\int_{\{\beta=-r\} \cap E} \mathrm{e}^{a \beta} \mathrm{e}^{-f}-\int_{\{\beta=-t\} \cap E} \mathrm{e}^{a \beta} \mathrm{e}^{-f} \\
& =\mathrm{e}^{-a r} A_{f}(\{\beta=-r\} \cap E)-\mathrm{e}^{-a t} A_{f}(\{\beta=-t\} \cap E),
\end{aligned}
$$

where we have used $|\nabla \beta|=1$. Here, $A_{f}(\Omega)$ denotes the area of the set $\Omega$ with respect to the weighted area form. This shows for a fixed $r$ and all $t>r$

$$
A_{f}(\{\beta=-t\} \cap E) \leq C(r) \mathrm{e}^{a t} .
$$

Integrating with respect to $t$ from $r$ to $R$ we obtain an upper bound for the volume of the sublevel sets of the Busemann function. Since the Busemann function is equivalent with the distance function on $E$, it follows that for $R>0$,

$$
V_{f}\left(B_{p}(R) \cap E\right) \leq C \mathrm{e}^{a R}
$$

Consider the function

$$
B:=\mathrm{e}^{\frac{1}{2} a \beta}
$$

By (4.2) and $|\nabla \beta|=1$, we have

$$
\Delta_{f} B \geq-\frac{1}{4} a^{2} B
$$

We now use $B$ as a test function for the variational formula of $\lambda_{1}\left(\Delta_{f}\right)=$ $\frac{1}{4} a^{2}$. For this sake, we define a cut-off function $\phi$ with support in $B_{p}(2 R)$ such that $\phi=1$ on $B_{p}(R)$ and $|\nabla \phi| \leq \frac{C}{R}$. Then, according to the variational 
characterization of $\lambda_{1}\left(\Delta_{f}\right)$, we have

$$
\begin{aligned}
\frac{1}{4} a^{2} & \int_{M}(B \phi)^{2} \mathrm{e}^{-f} \leq \int_{M}|\nabla(B \phi)|^{2} \mathrm{e}^{-f} \\
& =\int_{M}|\nabla B|^{2} \phi^{2} \mathrm{e}^{-f}+\frac{1}{2} \int_{M}\left\langle\nabla B^{2}, \nabla \phi^{2}\right\rangle \mathrm{e}^{-f}+\int_{M}|\nabla \phi|^{2} B^{2} \mathrm{e}^{-f} \\
& =\int_{M}|\nabla B|^{2} \phi^{2} \mathrm{e}^{-f}-\frac{1}{2} \int_{M}\left(\Delta_{f} B^{2}\right) \phi^{2} \mathrm{e}^{-f}+\int_{M}|\nabla \phi|^{2} B^{2} \mathrm{e}^{-f} \\
& =-\int_{M} B\left(\Delta_{f} B\right) \phi^{2} \mathrm{e}^{-f}+\int_{M}|\nabla \phi|^{2} B^{2} \mathrm{e}^{-f} \\
& =\frac{1}{4} a^{2} \int_{M} B^{2} \phi^{2} \mathrm{e}^{-f}+\int_{M}|\nabla \phi|^{2} B^{2} \mathrm{e}^{-f}-\int_{M} B\left(\Delta_{f} B+\frac{1}{4} a^{2} B\right) \phi^{2} \mathrm{e}^{-f}
\end{aligned}
$$

Therefore,

$$
\int_{M} B\left(\Delta_{f} B+\frac{1}{4} a^{2} B\right) \phi^{2} \mathrm{e}^{-f} \leq \int_{M}|\nabla \phi|^{2} B^{2} \mathrm{e}^{-f}
$$

From (4.1) and (4.3) and the construction of $B$, arguing as in [21], we have

$$
\int_{M}|\nabla \phi|^{2} B^{2} \mathrm{e}^{-f} \leq \frac{C}{R} \rightarrow 0
$$

In view of (4.4), we conclude $\Delta_{f} B+\frac{1}{4} a^{2} B=0$. In particular, $B$ is smooth. Therefore,

$$
\Delta_{f} \beta=-a \text { and }|\nabla \beta|=1
$$

hold everywhere on $M$. Now, by the Bochner formula,

$$
0=\frac{1}{2} \Delta_{f}|\nabla \beta|^{2}=\left|\beta_{i j}\right|^{2}+\left\langle\nabla \Delta_{f} \beta, \nabla \beta\right\rangle+R i c_{f}(\nabla \beta, \nabla \beta) \geq\left|\beta_{i j}\right|^{2}
$$

This proves $\beta_{i j}=0$, which implies $M$ is a direct product given by $M=$ $\mathbb{R} \times N$. That $N$ is compact follows from the assumption of $M$ having two ends. This proves the theorem.

We now discuss an important application of this result to the gradient Ricci solitons. Recall that a gradient steady Ricci soliton is a manifold $(M, g, f)$ such that $R_{i j}+f_{i j}=0$. Here we prove that gradient steady Ricci solitons are either connected at infinity or they are trivial, that is, isometric to a Ricci flat cylinder. 
Theorem 4.2. Let $(M, g, f)$ be a gradient steady Ricci soliton. Then either $M$ is connected at infinity or $M$ is isometric to $\mathbb{R} \times N$ for a compact Ricci flat manifold $N$.

Proof of Theorem 4.2. Let us assume that $M$ has at least two ends. It is known that for any gradient steady Ricci soliton there exists $a>0$ so that $|\nabla f|^{2}+S=a^{2}$, see (2.7). By Proposition 2.1 we know that $\lambda_{1}\left(\Delta_{f}\right)=\frac{1}{4} a^{2}$. Then, by Theorem 4.1 we conclude $M=\mathbb{R} \times N$ for some compact manifold $N$. It is easy to see $N$ has to be a steady Ricci soliton also. However, any compact steady gradient Ricci soliton must be Ricci flat. This concludes the proof.

\section{Acknowledgments}

The first author has been partially supported by NSF grant No. DMS1005484. Wang would like to thank Ben Chow for his interest in this work.

\section{References}

[1] D. Bakry and M. Émery, Diffusions hypercontractives. In Seminaire de probabilites, XIX, 1983/84, 177-206, Lecture Notes in Math. 1123 Springer, Berlin, 1985.

[2] K. Brighton, A Liouville-type theorem for smooth metric measure spaces, arXiv:1006.0751.

[3] P. Buser, A note on the isoperimetric constant, Ann. Sci. Ecole Norm. Sup. 15 (1982), 213-230.

[4] H.D. Cao, Recent progress on Ricci solitons, Adv. Lect. Math. 11(2) (2010), 1-38.

[5] I. Chavel, Riemannian Geometry - A Modern Introduction, 2nd en., Cambridge University Press, 2006.

[6] J. Cheeger, T. Colding and W. Minicozzi, Linear growth harmonic functions on complete manifolds with non-negative Ricci curvature, Geom. Funct. Anal. 5 (1995), 948-954.

[7] B. Chow, P. Lu and L. Ni, Hamilton's Ricci flow, Graduate Studies in Mathematics, 2006. 
[8] B.L. Chen, Strong uniqueness of the Ricci flow, J. Differential Geom. 82(2) (2009), 362-382.

[9] S.Y. Cheng, Eigenvalue comparison theorems and its geometric applications, Math. Z. 143 (1975), 289-297.

[10] S.Y. Cheng and S.T. Yau, Differential equations on Riemannian manifolds and their geometric applications, Comm. Pure Appl. Math. 27 (1975), 333-354.

[11] T. Colding and W. Minicozzi, Harmonic functions with polynomial growth, J. Differential Geom. 46 (1997), 1-77.

[12] F. Fang, X.-D. Li, and Z. Zhang, Two generalizations of CheegerGromoll splitting theorem via Bakry-Émery Ricci curvature, Ann. Inst. Fourier, 59(2) (2009), 563-573.

[13] A. Grigor'yan, The heat equation on noncompact Riemannian manifolds, Math. USSR Sbornik. 72 (1992), 47-77.

[14] P. Hajłasz and P. Koskela, Sobolev meets Poincaré, C. R. Acad. Sci. Paris Sr. I Math. 320 (1995), 1211-1215.

[15] R. Hamilton, The formation of singularities in the Ricci flow, Surv. Differential Geom. 2 (1995), 7-136, International Press.

[16] P. Li, Harmonic functions and applications to complete manifolds, lecture notes, available on math.uci.edu/pli.

[17] P. Li, Harmonic sections of polynomial growth, Math. Res. Lett. 4 (1997), 35-44.

[18] P. Li and L.-F. Tam, Linear growth harmonic functions on a complete manifold, J. Differential Geom. 29 (1989), 421-425.

[19] P. Li and J. Wang, Complete manifolds with positive spectrum, J. Differential Geom. 58 (2001), 501-534.

[20] P. Li and J. Wang, Complete manifolds with positive spectrum, II. J. Differential Geom. 62 (2002), 143-162.

[21] P. Li and J. Wang, Connectedness at infinity of complete Kähler manifolds, Amer. J. Math. 131 (2009), 771-817.

[22] X.D. Li, Liouville theorems for symmetric diffusion operators on complete Riemannian manifolds, J. Math. Pures Appl. 84 (2005), 1295-1361. 
[23] A. Lichnerowicz, Varietes riemanniennes a tensor $C$ non negatif. C. R. Acad. Sci. Paris Sr. A, 271 (1970) 650-653.

[24] O. Munteanu and N. Sesum, On gradient Ricci solitons, to appear in J. Geom. Anal.

[25] O. Munteanu and J. Wang, Analysis of the weighted Laplacian and applications to Ricci solitons, preprint.

[26] A. Naber, Some geometry and analysis on Ricci solitons, arXiv:math.DG/0612532.

[27] G. Perelman, The entropy formula for the Ricci flow and its geometric applications, arXiv:math.DG/0211159.

[28] L. Saloff-Coste, A note on Poincaré, Sobolev and Harnack inequalities, Int. Math. Res. Not. 2 (1992), 27-38.

[29] L. Saloff-Coste, Aspects of Sobolev-Type Inequalities, London Mathematical Society Lecture Notes Series, 2001

[30] R. Schoen and S.T. Yau, Lectures on Differential Geometry, International Press, 1994.

[31] G. Wei and W. Wylie, Comparison geometry for the Bakry-Émery Ricci tensor, J. Differential Geom. 83 (2009), 377-405.

[32] J. Wu, Upper bounds on the first eigenvalue for a diffusion operator via Bakry-Émery Ricci curvature II, arXiv: math.DG/1010.4175v1.

[33] N. Yang, A note on nonnegative Bakry-Émery Ricci Curvature, Arch. Math. 93(5) (2009), 491-496.

[34] S.T. Yau, Harmonic functions on complete Riemannian manifolds, Comm. Pure Appl. Math. 28 (1975), 201-228.

[35] P. Li and L.-F. Tam, Harmonic functions and the structure of complete monifolds, J. Differential Geom. 35 (1992), 359-383.

[36] P. Li, Large time behavior of the heat kernel on complete manifolds with non-negative Ricci curvature, Ann. Math. 124 (1986), 1-21.

Department of Mathematics

Columbia University

NEW YoRK, NY 10027

USA

E-mail address: omuntean@math.columbia.edu 
School of Mathematics

UNIVERSITY OF MiNNESOTA

MinNEAPOLIS, MN 55455

USA

E-mail address: jiaping@math.umn.edu

Received March 4, 2011 